\title{
Learning the Fructose Metabolism by Hypermedia
}

Sugai, J.K. ${ }^{1}$, Figueiredo, M.S.R.B. ${ }^{1}$; Antônio, R.V. ${ }^{2}$; Heidrich, D. ${ }^{1}$, Angotti, J.A.P. ${ }^{3}$ ${ }^{1}$ Departamento de Bioquímica, Centro de Ciências Biológicas, ${ }^{2}$ Campus de Araranguá, ${ }^{3}$ Departamento de Metodologia do Ensino, Centro de Ciências da Educação, Universidade Federal de Santa Catarina, Florianópolis, SC, Brazil.

Introduction: Learning of biochemistry among other factors depends on create an educational environment favorable to the reality of the students and offer better educational tools for the teachers. Objective: The goal of this hypermedia is to motivate and strengthen the students learning about the metabolism of fructose. Materials And Methods:This work presents the development of a biochemical hypermedia to understanding of the fructose metabolism. It was developed with the help of concept maps, ISIS Draw, ADOBE Photoshop and FLASH MX Program. Results and Discussion: A step by step animation process shows the enzymatic reactions of three pathways of the fructose metabolism (principal route-fructokinase, sorbitol and hexokinase pathways). There are navigation guide that allow scrolling the mouse over the names of the components of enzymatic reactions of via the metabolism of fructose. Thus, explanatory text box, chemical structures and animation of the actions of enzymes appear to navigator. Upon completion of the module, the user's response to the proposed exercise can be checked immediately through text box with interactive content of the answer. Conclusion:This hypermedia was presented for undergraduate students (UFSC). Its use allowed to users to reach the goal of understanding of the subject independently of their agility or background, since it was possible to each student navigate through the hypermedia according with his individual rhythm and as many times as needed. Interactive hypermedia is proving to be a valuable tool to improve and facilitate the understanding of biochemistry subjects.

Keywords: biochemical hypermedia, fructose metabolism, fructose pathway. Supported by: CNPq. 